\title{
TIC y el fortalecimiento de competencias matemáticas en estudiantes de pedagogía de la enseñanza matemática
}

\author{
TIC and the strengthening of mathematical competencies in mathematics education students \\ TIC e o fortalecimento de competências matemáticas em alunos de educação matemática
}

\section{Diego Zavala Urquizo \\ dzavala@uce.edu.ec \\ https://orcid.org/0000-0003-4883-922X}

Universidad Central del Ecuador, Quito-Ecuador

Karlita Muñoz Correa

kmunoz@uce.edu.ec

https://orcid.org/0000-0002-8695-7967

Universidad Central del Ecuador, Quito-Ecuador

\author{
Juan Cobos Velasco \\ jccobos@uce.edu.ec \\ https://orcid.org/0000-0002-9770-3727
}

Universidad Central del Ecuador, Quito-Ecuador

Guthnara Muñoz Correa

gkmunozc@uce.edu.ec

https://orcid.org/0000-0002-5979-6395

Universidad Central del Ecuador, Quito-Ecuador

Artículo recibido 19 de julio 2021, arbitrado y aceptado 13 de septiembre 2021 y publicado 13 de diciembre 2021

\section{RESUMEN}

El desarrollo continuo de las Tecnologías de la Información y Comunicación (TIC) se han convertido en una de las principales opciones para el mejoramiento de actividades generales y específicas de la sociedad. La presente investigación busca determinar cómo las TIC contribuyen a fortalecer el desarrollo de las competencias matemáticas en la formación del futuro docente de la Carrera de Pedagogía de las Matemáticas de la Universidad Central del Ecuador. Se entiende por competencia matemática aquellas habilidades y destrezas que permitan pensar, comunicarse y utilizar el lenguaje simbólico matemático, resolver problemas y utilizar herramientas y recursos. En tal sentido, se evalúo que competencias matemáticas puede fortalecer el futuro docente de esta área del conocimiento, su nivel de desarrollo y su aplicación en la práctica docente con el uso de los diversos recursos y herramientas que ofrecen las TIC. Para ello se efectuó un estudio cuantitativo de nivel bibliográfico, de campo, aplicada, descriptivo y tipo cuasi-experimental, el cual permitió concluir la incidencia de las TIC en el desarrollo de las competencias matemáticas básicas de los futuros docentes. En función al tema abordado dentro del artículo, debe señalar objetivo, metodología, resultados y conclusiones.

Palabras clave: TIC, Competencias matemáticas; Entornos virtuales de aprendizaje; Tecnología educativa; competencias digitales

\section{ABSTRACT}

The continuous development of Information and Communication Technologies (ICT) has become one of the main options for the improvement of general and specific activities of society. This research seeks to determine how ICT contribute to strengthening the development of mathematical skills in the training of future teachers of the Mathematics Pedagogy Career of the Central University of Ecuador. Mathematical competence is understood to be those skills and abilities that allow thinking, communicating and using mathematical symbolic language, solving problems and using tools and resources. In this sense, it was evaluated that mathematical competencies can strengthen the future teacher of this area of knowledge, its level of development and its application in teaching practice with the use of the various resources and tools offered by ICT. To this end, a quantitative study was carried out at the bibliographic, field, applied, descriptive and quasi-experimental level, which allowed to conclude the incidence of ICT in the development of basic mathematical skills of future teachers. Depending on the topic addressed within the article, it must indicate objective, methodology, results and conclusions.

Key words: TIC; mathematical competencies; Virtual learning environments; Educational technology; Digital competencies

\section{RESUMO}

O desenvolvimento contínuo das Tecnologias da Informação e Comunicação (TIC) tornaram-se uma das principais opções para o melhoramento de atividades gerais e específicas da sociedade. A presente pesquisa busca determinar como as TIC contribuem para fortalecer o desenvolvimento das competências matemáticas na formação do futuro docente da carreira de Pedagogia das Matemáticas da Universidade Central do Equador. Entende-se por competência matemática aquelas habilidades e destrezas que permitam pensar, comunicar e utilizar a linguagem simbólica matemática, resolver problemas e utilizar ferramentas e recursos. Nesse sentido, avaliou-se que competências matemáticas podem fortalecer o futuro docente desta área do conhecimento, seu nível de desenvolvimento e sua aplicação na prática docente com o uso dos diversos recursos e ferramentas que oferecem as TIC. Para isso, realizou-se um estudo quantitativo de Nível bibliográfico, de campo, aplicado, descritivo e tipo quase-experimental, o qual permitiu concluir a incidência das TIC no desenvolvimento das competências matemáticas básicas dos futuros docentes. com base no tópico abordado dentro do artigo, você deve apontar objetivo, Metodologia, resultados e conclusões.

Palavras-chave: TIC; competências matemáticas; Ambientes de aprendizagem virtual; Tecnologia educativa; competências digitais 


\section{INTRODUCCIÓN}

El desarrollo acelerado de las Tecnologías de la Información y Comunicación (TIC) se ha convertido en una de las principales opciones para el mejoramiento en actividades generales y específicas de la sociedad actual. Su aplicabilidad en diversos procesos de cotidianidad están enfocados en la mejora y optimización del tiempo y esfuerzo para su cumplimiento, estas características han convertido a las TIC en un elemento esencial de cambio global. La integración de las TIC está transformando nuestra sociedad en todos los ámbitos, entre ellos la cultura científica, base sobre la cual se instaura el desarrollo de la sociedad moderna (Colás y De Pablos, 2012).

La integración de las TIC en el sistema nacional de educación (SNE) de Ecuador, es un proceso que denota algunas deficiencias en su aplicación, entre estas se tiene el desarrollo incompleto de las competencias digitales por parte de los docentes, requisito básico para su aplicación en el aula (Fernández-Cruz y Fernández-Díaz, 2016). La ausencia de un proyecto estandarizado en el (SNE), sobre el uso e integración de las TIC, puede generar como resultado que su aplicación didáctica no muestre en su totalidad aspectos de innovación o mejora en contraposición a las prácticas tradicionales de enseñanza (Christian y Mathrani, 2014). Es por esto que uno de los puntos claves de la transformación educativa debe ser la capacitación docente en estrategias de enseñanza e integración de las tecnologías en el proceso educativo (Caccuri, 2014).

Estudios realizados en los últimos años (Díaz, 2010; Morales, 2012; Avecilla, Cárdenas y Barahona, 2015; Cárdenas y González, 2016), han dedicado sus esfuerzos a destacar el aporte académico frente a la consolidación de espacios pedagógicos significativos en el fortalecimiento de las competencias y habilidades, para un desarrollo cognitivo y metacognitivo profundo. De este modo, aparecen las competencias matemáticas como elementos fundamentales en el desarrollo de un pensamiento lógico y la apropiación del avance científico y tecnológico (MEN, 2006); donde, sin duda las TIC contribuyen en cada uno de sus procesos asociados, estas investigaciones muestran conclusiones de incidencia positiva direccionada al uso y aplicación de herramientas TIC como; entornos virtuales de aprendizaje, software específico, objetos virtuales de aprendizaje e internet enfocados en procesos de enseñanza y aprendizaje de las matemáticas.

En este contexto, otros estudios como los de Martínez, León y Villegas, (2011); Moreno, (2007); Parra y Díaz, (2014) abordan el tema de las competencias básicas del docente, la didáctica de la matemática y las TIC, con un enfoque integrador en los procesos educativos, mismos que buscan examinar el uso efectivo de la tecnología y cuáles son los niveles de dominio de estas competencias mediante una aproximación tecno-pedagógica a la sociedad, que basa su desarrollo y crecimiento económico en diversos factores, entre estos las matemáticas y la tecnología.

De igual manera, el nivel de desarrollo de las competencias matemáticas planteadas por el Programa Internacional de Evaluación de los Alumnos (PISA) y su fortalecimiento mediante el uso y aplicación de las TIC. Para poder determinarlo se hace énfasis en la importancia de la evaluación de sistemas y procesos educativos, es así que evaluaciones nacionales e internacionales se han 
instaurado en el SNE, con la finalidad de obtener respuestas para el mejoramiento de las políticas socioeducativas a nivel curricular y económico; es por esta situación que en Ecuador se creó en Instituto Nacional de Evaluación Educativa (INEVAL), ente que a través de su Consejo Directivo ha logrado incorporar a Ecuador en el Proyecto PISA que engloba evaluaciones en las áreas de lectura, matemática y ciencias. Dichas evaluaciones no se basaron en su totalidad en el dominio del currículo escolar, sino en las destrezas y conocimientos necesarios para la vida adulta, así como en el nivel de competencias específicas en estas áreas. Los resultados de estas evaluaciones tienen la finalidad y compromiso de dar a conocer las falencias del SNE y poder realizar propuestas para incrementar la calidad educativa, con el objetivo de convertir a la educación en un factor clave de desarrollo personal y social en la nación.

Paratal efecto,eldesarrolloóptimo del procesode enseñanza y aprendizaje se fundamenta en aspectos didácticos, pedagógicos y curriculares, siendo estos la base fundamental para su consecución. En la actualidad se puede adicionar a la tecnología como parte de estos aspectos, sin dejar de preguntarnos, ¿cómo inciden las TIC a los procesos educativos? Esta pregunta se planteó debido a factores propios de las TIC, como la masividad, accesibilidad, portabilidad y la ubicuidad, esta situación obliga a buscar alternativas que nos permitan realizar un proceso de integración de manera adecuada, con la finalidad de que estas prácticas tengan la incidencia necesaria para poder ser consideraras significativas.

Como manifiesta Bisquerra (2004), identificar el problema de investigación plantea mejorar y definir formalmente la idea inicial de una investigación con la finalidad de comprender mejor algún suceso. En este sentido al plantear las problemáticas que abordan esta investigación se podría iniciar con la identificación del poco desarrollo de las competencias, denotado en los resultados obtenidos por Ecuador en el proceso de evaluación del proyecto PISA, y la carencia de estrategias didáctico-pedagógicas en los procesos áulicos de la carrera de pedagogía de la enseñanza Matemática, es en este contexto se debe buscar las estrategias adecuadas para el mejoramiento de la calidad de la educación, tomando en cuenta que el docente al ser una parte fundamental de este proceso deberá tener acciones vinculantes con su formación continua en temas relacionados al uso, aplicación e integración de las TIC en los procesos educativos, con la finalidad de garantizar su desempeño docente de manera óptima en su labor profesional.

\section{Fundamentación teórica}

Las universidades tienen la responsabilidad de integrar en sus procesos de enseñanza y aprendizaje estrategias creativas e innovadoras, en vista de que, en la sociedad del conocimiento, los valores y prácticas de creatividad e innovación desempeñan un papel importante para responder mejor a las nuevas necesidades del entorno social y educativo. Es así que en este ambiente las personas deben hacer uso de la información con principios éticos y responsables, en medio de una gran cantidad de información y herramientas tecnológicas, sin olvidar el espíritu crítico y el ejercicio de las capacidades cognitivas que permitan diferenciar la información importante de la que no lo es.

Este contexto es particularmente importante en el caso de la formación del futuro docente por cuanto debe desarrollar competencias específicas, de acuerdo con su especialidad, para poder 
ejercerla de forma pertinente. En el caso particular de la formación del futuro docente de matemática, especialidad que puede reforzar los procesos de enseñanza y aprendizaje con el uso didáctico de software específico, objetos virtuales de aprendizaje (OVAS) y simuladores, se plantea como las TIC fortalecen el desarrollo de las competencias matemáticas.

Es así que la educación basada en competencias es un enfoque que evidencia el aprendizaje de conocimientos, el desarrollo de habilidades, actitudes y comportamientos requeridos, para el desempeño de un papel específico, para capacitarse en el estudio de una profesión o para realizar adecuadamente una tarea determinada (Bambozzi y Vadori, 2009).

La definición que propone Tobón (2013), y que se ha debatido con expertos en diversos escenarios, publicaciones y congresos, es que las competencias son procesos complejos de desempeño con idoneidad en un determinado contexto, con responsabilidad. Se puede considerar que esta definición trata del desempeño y responsabilidad, reúne los atributos generales de la competencia: conocimiento, habilidad y actitud. Cualquier concepto de competencias que comprenda esos tres atributos podría ser funcional y aplicable a la cotidianidad.

La idoneidad de las competencias se enfoca en realizar actividades, cumpliendo criterios de calidad como la eficacia, eficiencia, efectividad, pertenencia y aprobación establecidos de forma particular. Los contextos constituyen el campo disciplinar, social, cultural y ambiental que rodean e influyen en determinada situación (Tobón, 2013).
En la misma línea Perronoud (2004), propone la caracterización de las competencias como un conjunto de acciones o decisiones que un individuo puede tomar para resolver en un determinado contexto; en este sentido, se plantea que ser competente involucra una acción para transformar el saber en poder. Llegar a ser competente implica el dominio de un saber y así mismo disponer de las estrategias adecuadas para poner en práctica ese saber y conseguir con éxito la resolución de una tarea en un contexto concreto.

En este sentido se debe tomar en cuenta que la competencia matemática es fundamental para avanzar en los diferentes niveles educativos. En este estudio se consideran algunas definiciones que permitan tener en claro esta definición: Para Niss (2002) dominar las matemáticas quiere decir poseer competencia matemática y se interpreta como la capacidad de entender, juzgar, hacer y usar las matemáticas en una variedad de contextos y situaciones de la cotidianidad.

Por su parte, para Guzmán, Obonaga y Gutiérrez (2015), la competencia matemática permite "la interrelación de componentes cognitivos, procedimentales y actitudinales, que ayudan a los estudiantes a dar respuesta a los problemas a los que se enfrenten" (p.2). Por consiguiente, los autores mencionados plantean que la magnitud de la competencia en las personas se muestra cuando se enfrentan a un evento complejo, ya que recalcan "más en lo que el estudiante puede hacer con sus conocimientos y habilidades, que en el dominio de los mismos" (p. 2).

Para Rico y Lupiañez (2009) en su obra "Competencias matemáticas desde una perspectiva 
curricular", mencionan las siguientes competencias enmarcadas como fundamentales en el proyecto PISA: Pensar y razonar: constituye la forma en que se plantea y se da respuesta a cuestiones propias de la matemática, su capacidad para desarrollar estrategias de resolución independientes $\mathrm{o}$ guiadas, así como la generación del razonamiento matemático evidente y avanzado. Argumentar y justificar: representa el modo en que el estudiante justifica sus afirmaciones acerca del conocimiento matemático. Esta justificación y proposición se enlaza en forma de demostraciones matemáticas, mismas que se convierten en herramientas que permiten validar el conocimiento matemático. Comunicar: está enfocada a las expresiones orales o escritas de las matemáticas y su comprensión e interpretación a enunciados orales o escritos propuestos por otras personas. Modelizar: se enmarca en la estructuración y análisis de un problema inicial con rigor matemático que genere los procesos que le permitan al estudiante el uso y manejo del conocimiento matemático para la resolución de problemas. Plantear y resolver problemas: permite al estudiante estar en la capacidad de plantear, formular y definir diferentes problemas matemáticos: puros, aplicados, de respuesta abierta o cerrada, y que su resolución se pueda dar mediante una diversidad de estrategias de resolución. Representar: su interpretación es una relación directa para decodificar y codificar, interpretar y distinguir entre los diferentes tipos de representación de objetos matemáticos, además de poder escoger y relacionar diferentes formas de representación de acuerdo con la situación y el propósito planteado. Utilizar lenguaje simbólico, formal y técnico, y las operaciones: Partiendo de su relación con la competencia representar, es el complemento para que los estudiantes puedan decodificar, interpretar y relacionar el lenguaje simbólico y formal con el lenguaje natural, llegando a utilizar variables, resolver ecuaciones y comprender cálculos con un manejo apropiado de enunciados y expresiones. Emplear herramientas y soportes tecnológicos: Se centra en el uso de recursos TIC que contribuyan a la resolución de problemas matemáticos. Esta competencia se expresa en el conocimiento de posibilidades, capacidad de uso $\mathrm{y}$ aplicación fundamentada en los beneficios y limitaciones de cada herramienta o recurso web.

En relación con la temática expuesta, se tiene la investigación de Belfori (2014), quien indica que los recursos TIC contribuyen a los procesos didácticos de información, colaboración y aprendizaje en el campo de la formación docente, enfatizando la importancia del uso y aplicación de las TIC en la construcción del conocimiento por parte de los estudiantes, sin descuidar el dominio del contenido, la pedagogía y la tecnología; aristas que se fundamentan como fuente teórico conceptual de la presente investigación para determinar la incidencia de las TIC en el desarrollo de las competencias matemáticas de los futuros docentes.

En este sentido, el estudio se fundamentó en cómo las TIC inciden en el desarrollo de las competencias matemáticas, para ello, se formularon los objetivos establecer el grado de rendimiento académico del grupo experimental en relación con el grupo control, en el desarrollo de las competencias matemáticas con la aplicación de las TIC en los procesos áulicos con el fin de determinar la incidencia de las TIC en los procesos de desarrollo de competencias matemáticas. 
MÉTODO

El planteamiento metodológico de esta investigación tuvo un enfoque cuantitativo, de nivel bibliográfico, de campo, aplicado, descriptivo y tipo cuasi-experimental el cual permitió por medio de tres instrumentos, en el que se identificó dos dimensiones: TIC, y Competencias matemáticas con el objetivo de determinar la incidencia de las TIC en el desarrollo de las competencias matemáticas básicas de los futuros docentes, este proceso se realizó con la aplicación de los instrumentos a dos grupos de estudiantes con características similares; denominados grupo control y grupo experimental, con quienes se realizó actividades pedagógicas de tipo tradicional y no tradicional con el uso de las TIC, durante 12 semanas, tiempo en el que se realizaron mediciones evaluativas de tipo diagnóstica, formativa y sumativa, que permitieron comparar el rendimiento académico obtenido por cada grupo y así poder determinar el nivel de incidencia que tienen las TIC en el fortalecimiento de las competencias matemáticas.

Paralaejecución delainvestigación, seseleccionó como población objetivo a los estudiantes de la carrera de pedagogía de la enseñanza Matemática, se determinó la muestra óptima correspondiente a los diferentes instrumentos aplicados; obteniéndose un total de 80 estudiantes divididos dos grupos (control y experimental) a dicha muestra de 80 estudiantes se aplicó como instrumento de recolección de datos tres cuestionarios divididos en tres etapas; Etapa 1: Evaluación Diagnóstica Pre-test, Etapa 2: Evaluación Formativa Post-test de seguimiento y Etapa 3: Evaluación Sumativa Post-test.

Además, el método de muestreo que se utilizó para la aplicación de los instrumentos fue el probabilístico-aleatorio estratificado por estudiantes de tercer semestre de la Carrera de pedagogía de la enseñanza Matemática.

En este sentido, la población considerada en la investigación correspondió a los estudiantes de tercer semestre de la carrera de pedagogía de la enseñanza Matemática con un total de 100 estudiantes, además se enmarcó en un trabajo interdisciplinario entre los docentes de las asignaturas Matemática Estructurada y Tecnologías de la Información y Comunicación, luego de determinar la muestra igual a 80 estudiantes, la metodología de trabajo inició determinando los paralelo Ay B de forma aleatoria, el grupo de control fue el paralelo A y el grupo experimental fue el paralelo B. Los estudiantes iniciaban tercer semestre en la Carrera, de los cuales ninguno de los grupos contaba con estudiantes repetidores de la materia, su particularidad principal estuvo determinada por que era la primera ocasión que tomaría esta asignatura. Una vez determinada esta situación inicial se procedió a cumplir con los planes y programas planificados por el docente responsable de la asignatura. Se asignó dos estudiantes pasantes que fueron ubicados uno en cada paralelo con la función de brindar su contingente y soporte al proceso áulico, el estudiante pasante del grupo experimental tuvo como tarea ayudar al docente con soporte tecnológico para los procesos de enseñanza y aprendizaje.

El docente responsable de la asignatura procedió durante esta planificación, que duró 12 semanas, a impartir clases con metodología tradicional en el grupo control y con el uso de las TIC en el grupo experimental. Cabe señalar que se inició con una evaluación diagnóstica, en la cual se pudieron determinar los prerrequisitos en los estudiantes; luego de la quinta semana se procedió 
a registrar la segunda evaluación en este caso de tipo formativa, misma que permitió ir dilucidando la captación de los tópicos trabajados en clase con base a las dos metodologías de enseñanza aplicadas; para finalizar, en la doceava semana, se procedió a registrar la tercera evaluación de tipo sumativa en la cual se pudieron determinar los niveles de aprendizaje de la asignatura. Para las evaluaciones se produjo una batería de treinta preguntas con la guía de los compañeros del área de matemática y los estudiantes del último semestre de la carrera (noveno semestre), las preguntas estaban distribuidas con base a los contenidos planificados. Con esta batería de preguntas se crearon tres cuestionarios para los diferentes momentos de evaluación explicados anteriormente, cada cuestionario conto con seis preguntas, alineadas al manual para elaboración de ítems publicado por el INEVAL López et al., (2013). Esta evaluación ayudó a determinar, con base a las medias, desviación estándar y nivel de significación de las calificaciones, si la hipótesis planteada y fundamentada en la inserción de las TIC en las actividades docentes fue o no efectiva.

\section{RESULTADOS Y DISCUSIÓN}

Con la aplicación de los instrumentos en sus diferentes momentos (diagnóstico, formativo y sumativo) se realizó un análisis descriptivo univariabledelos datos, el primerbloquecorresponde a datos informativos de los estudiantes; en el segundo bloque se planteó los ítems relacionados con las competencias matemáticas, aplicadas a las unidades temáticas de ecuaciones lineales y cuadráticas, para el proceso de recolección de datos, se determinó la calificación correspondiente para cada uno de los cuestionarios igual a 6 puntos. Se organizó la información de los valores de las calificaciones obtenidas por los respectivos grupos de aplicación (control y experimental), en tablas de información. Se utilizó el programa SPSS, para procesar las tablas de información que resumen los valores obtenidos en los diferentes instrumentos de evaluación, determinando la frecuencia y luego los respectivos cálculos descriptivos; media aritmética y desviación estándar. Se analizaron los datos obtenidos en términos descriptivos, con la finalidad de interpretarlos y responder a los objetivos de la investigación. Se confrontaron los hallazgos obtenidos con la teoría.

A continuación, se presenta la información obtenida por los grupos en los que se aplicaron las evaluaciones de la asignatura matemática estructurada, en los que se destacan las medidas descriptivas; media aritmética y desviación estándar, dichas medidas permitieron determinar el desarrollo y asimilación de los temas planificados, con las metodologías planteadas tradicional y con aplicación de las TIC, durante los procesos áulicos. 
Tabla 1. Registro de los valores estadísticos obtenidos por los grupos de aplicación en los diferentes momentos de evaluación.

\begin{tabular}{clcccc}
\hline \multirow{2}{*}{ No. } & Evaluaciones & \multicolumn{2}{c}{ Grupo control } & \multicolumn{2}{c}{ Grupo experimental } \\
& $\begin{array}{c}\text { Media } \\
\text { aritmética }\end{array}$ & $\begin{array}{c}\text { Desviación } \\
\text { estándar }(\boldsymbol{\sigma})\end{array}$ & $\begin{array}{c}\text { Media } \\
\text { aritmética }\end{array}$ & $\begin{array}{c}\text { Desviación } \\
\text { estándar }(\boldsymbol{\sigma})\end{array}$ \\
\hline 1 & Evaluación diagnóstica & 5.15 & 0.64 & 5.13 & 1.18 \\
2 & Evaluación formativa & 4.25 & 0.92 & 5.72 & 0.82 \\
3 & Evaluación sumativa & 4.66 & 0.81 & 5.91 & 0.78 \\
& PROMEDIO & $\mathbf{4 . 6 9}$ & $\mathbf{0 . 7 9}$ & $\mathbf{5 . 5 9}$ & $\mathbf{0 . 9 3}$ \\
\hline
\end{tabular}

En la Tabla 1, se inicia con la comparación de resultados obtenidos de la media aritmética de la evaluación diagnóstica aplicada a los grupos experimental y de control, se puede concluir que con las puntuaciones obtenidas, los dos grupos tienen el conocimiento necesario, para comenzar el estudio de la temática planteada, continuando con el segundo momento evaluativo (formativa) se puede observar que la diferencia entre los rendimientos de los grupos es de 1.47 puntos, diferencia que marca el grado de asimilación y rendimiento en la temática planteada, para finalizar en el último momento de evaluación (sumativa), el grupo experimental obtuvo mejores resultados en el rendimiento académico, con una diferencia moderada de 1.25 puntos. Para finalizar se realizó un promedio de los tres momentos evaluativos de estas 12 semanas, en el que se determinó que el grupo experimental obtuvo un porcentaje del $93.1 \%$ y el grupo control obtuvo un porcentaje del $78.1 \%$, determinando que el grupo experimental desarrollo de mejor forma las competencias matemáticas, debido al uso y aplicación de las TIC en los procesos áulicos planificados.

\section{Análisis y prueba de hipótesis general}

Referente a la relación de las dimensiones TIC y competencias matemáticas, en estudiantes de pedagogía de la enseñanza matemática, aplicada del grupo control y grupo experimental, correspondiente a los instrumentos de evaluación (diagnóstica, formativa y sumativa) en los diferentes momentos de estudio, se contrasto las siguientes hipótesis:

H0: El uso y aplicación de las TIC no inciden en el fortalecimiento de las competencias matemáticas, en los estudiantes de la Carrera de Pedagogía de la enseñanza matemática.

H1: El uso y aplicación de las TIC inciden en el fortalecimiento de las competencias matemáticas, en los estudiantes de la Carrera de Pedagogía de la enseñanza matemática.

Para determinar el cálculo de homogeneidad de la varianza se utilizó la prueba Levene y para aprobar o rechazar la hipótesis se aplicó la prueba $t$ para igualdad de medias. 
Para realizar prueba de hipótesis se eligió la prueba estadística de distribución normal Z, que se denota con $\mathrm{Z}$ o simplemente $\mathrm{Zc}$ al valor crítico, que separa las áreas de rechazo y aceptación de la hipótesis nula, para un nivel de significación del $5 \%, \alpha=0,05$.

También, se utilizó la prueba de Levene que determinó el supuesto de homogeneidad de varianzas, que considera que la varianza es constante en los diferentes niveles de un factor, es decir, entre diferentes grupos, esta prueba se utiliza a menudo antes de realizar una comparación de medias.
Finalmente, se utilizó la prueba t para igualdad de medias la cual es una prueba paramétrica que compara dos muestras independientes, mismas que deben cumplir características: asignación aleatoria de los grupos, homogeneidad de las varianzas de la variable dependiente de los grupos, distribución normal de la variable dependiente en los dos grupos.

A continuación, se presenta los resultados de los estudios de la prueba Levene y $\mathrm{t}$ :

Tabla 2. Estudio prueba de Levene, cálculo de homogeneidad de varianzas evaluación diagnóstica, formativa y sumativa, y prueba t para igualdad de medias

\begin{tabular}{|c|c|c|c|c|c|c|c|c|c|c|}
\hline \multirow{3}{*}{\multicolumn{2}{|c|}{ Tipo de Evaluación }} & \multicolumn{4}{|c|}{$\begin{array}{l}\text { Prueba de Levene de } \\
\text { calidad de varianzas }\end{array}$} & \multicolumn{5}{|c|}{ Prueba t para la igualdad de medias } \\
\hline & & \multirow{2}{*}{ F } & \multirow{2}{*}{ sig. } & \multirow{2}{*}{ t } & \multirow{2}{*}{ Gl } & \multirow{2}{*}{$\begin{array}{c}\text { sig. } \\
\text { (bilateral) }\end{array}$} & \multirow{2}{*}{$\begin{array}{l}\text { Dif. de } \\
\text { medias }\end{array}$} & \multirow{2}{*}{$\begin{array}{l}\text { Dif de error } \\
\text { estándar }\end{array}$} & \multicolumn{2}{|c|}{$\mathbf{9 5 \%}$ de I C de la dif } \\
\hline & & & & & & & & & Inferior & Superior \\
\hline \multirow[t]{2}{*}{ Diagnóstica } & \multirow{6}{*}{ 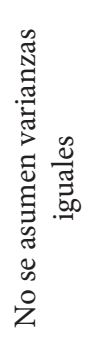 } & 23,920 & 000 & 2,573 & 78 & 0,012 & 0,30208 & 0,11738 & 0,06839 & 0,53578 \\
\hline & & & & 2,314 & 44,096 & 0,025 & 0,30208 & 0,13052 & 0,03905 & 0,56512 \\
\hline Formativa & & 5,706 & 019 & 1,223 & 78 & 0,225 & 0,11458 & 0,09365 & 0,07187 & 0,30103 \\
\hline & & & & 1,177 & 57,653 & 0,244 & 0,11458 & 0,09734 & 0,08030 & 0,30946 \\
\hline \multirow[t]{2}{*}{ Sumativa } & & 10,865 & 001 & 1,584 & 78 & 0,117 & 0,10417 & 0,06578 & 0,02678 & 0,23511 \\
\hline & & & & 1,347 & 35,898 & 0,186 & 0,10417 & 0,07732 & $-0,05266$ & 0,26099 \\
\hline
\end{tabular}

Los resultados obtenidos se muestran en la Tabla 2, donde el p-valor asociado al estadístico de contraste (Sig. $=0,117)$ de la variable evaluación sumativa es mayor que $0,05 \mathrm{y}$, el p-valor asociado al estadístico de contraste $($ Sig. $=0,225)$ de la variable evaluación formativa es mayor que 0,05 luego, al nivel de significación 0,05 no se tiene evidencias para rechazar la hipótesis nula, y el p-valor asociado al estadístico de contraste $($ Sig. $=0,012)$ de la variable evaluación diagnóstica es menor que 0,05 , esto significa que a nivel $\alpha=0,05$, se rechazó la hipótesis nula y se aceptó la hipótesis alternativa en la cual el uso y aplicación de las TIC incide en el fortalecimiento de las competencias matemáticas, en los estudiantes de la carrera de pedagogía de la enseñanza matemática.

Dado que en la distribución observada no hubo diferencia estadísticamente significativa, se aceptó que la media de las evaluaciones sumativa, formativa y diagnóstica del grupo control y grupo experimental, relacionadas con las competencias matemáticas adquiridas por los estudiantes es 
la misma o que la diferencia entre las medias aritméticas contiene el cero.

Mientras que en la distribución observada es estadísticamente significativa, se acepta que a nivel muestral existen diferencias entre las medias aritméticas de los dos grupos: control y experimental relacionada con las evaluaciones sumativa, formativa y diagnóstica; esto es, que las competencias matemáticas adquiridas por los estudiantes, en estas etapas, son diferentes.

\section{Discusión}

En concordancia con Paredes y Sanabria (2015), quienes manifiestan que el avance de las TIC y la virtualización, han abierto un escenario en el que se pueden desarrollar ambientes de aprendizaje, que facilitan el proceso de enseñanza $\mathrm{y}$ aprendizaje y permiten el acceso a un mayor número de personas, en el contexto de la presente investigación se observó que las prácticas cotidianas del aula al aplicar una metodología tradicional en el grupo control evidenció un menor rendimiento académico frente al grupo experimental de 15\%. Se determina que en procesos de enseñanza y aprendizaje del área del conocimiento de la matemática la utilización de las TIC desarrolla las competencias matemáticas en: Pensar y razonar, Argumentar y justificar, Comunicar, Modelizar, Plantear y resolver problemas, Representar, Utilizar lenguaje simbólico, formal y técnico, y las operaciones, Emplear herramientas y soportes tecnológicos generando espacio de mediación para la resolución de problemas en el contexto académico y la cotidianidad.

De acuerdo a la propuesta realizada por el Ministerio de Educación Nacional de Colombia MEN (2006), de "diseñar ambientes creativos de aprendizaje con mediación de TIC, para la enseñanza innovadora de Matemáticas, derivados de la sistematización de proyectos de aulas" (p.153), en el estudio realizado se pudo visualizar que la aportación de las TIC beneficia las prácticas para la enseñanza de las matemáticas, permitiendo que el estudiante genere su creatividad a través de los contenidos programáticos en la práctica por medio de espacios virtuales y herramientas que le permitan poner en práctica los procesos teóricos.

En concordancia con lo expresado por Belfori (2014), quien indica que los recursos TIC contribuyen a los procesos didácticos de información, colaboración y aprendizaje en el campo de la formación docente, nuestra investigación determinó que la utilización de la tecnología en espacios de aprendizaje virtual permite generar diversas herramientas didácticas, que permiten estimular los procesos de aprendizaje de los estudiantes dándoles la posibilidad de mantener un conocimiento perdurable en el tiempo y a resolver problemas del contexto educativo en cualquier área del conocimiento, haciéndoles competentes como profesores en el área de matemática en las diversas instituciones de educación secundaria del país.

\section{CONCLUSIONES}

En la investigación se evidenció que la utilización de las TIC en el desarrollo de las competencias matemáticas, tuvieron una incidencia positiva en el grupo experimental, misma que se diferencia en un $15 \%$, con respecto al grupo control, lo que permite concluir que la utilización de herramientas tecnológicas, entornos virtuales de aprendizaje y software específico en procesos de aprendizaje desarrollan las competencias matemáticas y permiten al estudiante reaprender la 
forma de plantear y resolver problemas propios de esta área del conocimiento.

En referencia a la evaluación aplicada en los tres momentos (inicial-diagnóstica; seguimientoformativa; y final-sumativa), arrojó como resultado que, en la evaluación sumativa la incidencia de uso y aplicación de las TIC es moderado; en la que se apreció la diferencia de los resultados en el rendimiento académico del grupo control igual a 4.69/6 puntos y el grupo experimental igual a 5.59/6 puntos, estableciendo una diferencia de 1.25 puntos a favor del grupo experimental. Por consiguiente, se concluyó que existe una relación directa entre las TIC y las competencias matemáticas para mejorar procesos de razonamiento, cognitivos y prácticos, y la comprensión de fundamentos que soportan el razonamiento abstracto y el pensamiento crítico de los estudiantes.

Bajo este contexto es necesario promover espacios virtuales de aprendizaje, articulados por los entornos virtuales, que se constituyen como herramientas dinámicas y afines con la aptitud $\mathrm{y}$ actitud de la juventud actual, debido a que los jóvenes universitarios han mostrado un apego a las TIC, a partir de las cuales se logra un verdadero aprendizaje significativo, que no está dado en función de la cantidad de conocimientos sino más bien de cómo saberlos utilizarlos en la cotidianidad.

\section{REFERENCIAS}

Avecilla, F., Cárdenas, O., y Barahona, B. (2015). GeoGebra para la enseñanza de la matemática y su incidencia en el rendimiento académico estudiantil. Revista Tecnológica ESPOL - RTE

Bambozzi, E y Vadori, G. (2009). Competencias Genéricas. Colección Cuadernos de Investigación. Argentina: Universitaria de la Universidad de Villa María
Belfori, L (2014). Uso del marco TPACK por estudiantes de un profesorado de matemática. Comité Latinoamericano de Matemática Educativa. 103(1), 1733-1740. Recuperado de https://core.ac.uk/display/33252559? recSetID=

Bisquerra, R. (2004). Metodología de la investigación educativa (Vol. 1). Editorial La Muralla. Recuperado de https://books.google. es/books?id= VSb4_cVukkcC\&lpg=PA3\&dq= B i s qu e r r a \% $202004 \& 1$ r \& h l= es \&pg $=$ PA $3 \# v=$ on epage $\& q B$ is querra $\% 20$ $2004 \& \mathrm{f}=$ false

Cárdenas, C., y González, D. (2016). Estrategia para la resolución de problemas matemáticos desde los postulados de Polya mediada por las TIC, en estudiantes del grado octavo del Instituto Francisco José de Caldas. Bogotá: Universidad Libre

Colás, P. y De Pablos, J. (2012). Aplicaciones de las tecnologías de la información y la comunicación en la investigación cualitativa. Revista española de pedagogía, 70(251), 77-92

Christian, S. y Mathrani, A. (2014). ICT Education: Socio-Learning Issues Faced by International Students. Proceedings of the Thirty Fifth International Conference on Information Systems, Auckland, New Zealand

Caccuri, V. (2014). Recursos TIC. Buenos Aires: Dalaga

Díaz, V. (2010). Procesos de simulación y modelación de situaciones problémicas de Cálculo Integral en ambientes dinámicos. En V Congreso Iberoamericano de Cabri (p.78-79). Querétaro, México: Universidad Autónoma de Querétaro

Fernández-Cruz, F. J. y Fernández-Díaz, M. J. (2016) Los docentes de la Generación Z y sus competencias digitales. Comunicar, 46(24), 97 105

Guzmán, C., Obonaga, G. y Gutiérrez, O. (2015). Competencias matemáticas, diseño y selección de tareas para el aprendizaje de las matemáticas en ingeniería. Presentado en la XIV Conferencia interamericana de educación matemática, 
CIAEM,Chiapas, México. Recuperado de: http://xiv.ciaem-redumate.org/index.php/xiv_ ciaem/xiv_ciaem/paper/viewFile/246/138

López, A., Sánchez, H., Carmona, M., et al. (2013). Elaboración de ítems de opción múltiple. Instituto Nacional de Evaluación Educativa. Quito, Ecuador

Martínez, J., León, O., y Villegas, Z. (2011). Competencias básicas que posee el docente de matemática en el ámbito de las tecnologías de información y comunicación (tic) en educación media técnica del distrito escolar no 4 de Guacara. Revista de Tecnología de Información y Comunicación En Educación, Vol. 51(1), 119132. Recuperado de http://servicio.bc.uc.edu. ve/educacion/eduweb/

Niebles, W., Martínez-Bustos, P., y Niebles-Núñez, L. (2019). Competencias matemáticas como factor de éxito en la prueba pro en universidades de Barranquilla, Colombia. Educación Y Humanismo, 22(38). https://doi.org/10.17081/ eduhum.22.38.3590

MEN. (2006). Estándares básicos de competencias en matemáticas, potenciar el pensamiento matemático: ¡un reto escolar! Bogotá: Ministerio de Educación Nacional. Recuperado de https://www.mineducacion.gov.co/1621/ articles-116042_archivo_pdf2.pdf

MEN. (2016). Matriz de Referencia matemáticas. Siempre Día-E la ruta hacia la excelencia educativa. Ministerio de Educación Nacional. Recuperadodehttp://aprende.colombiaaprende. edu.co/ckfinder/userfiles/files/articles-352712_ matriz_m.pdf

Morales, C.A. (2012). El desarrollo del pensamiento espacial y la competencia matemática. Una aproximación desde el estudio de los cuadriláteros. Revista Amazonia Investiga / Florencia, Colombia, 1 (1): 54-81p

Moreno, M. (2007). De la matemática formal a la matemática escolar. Revista de Investigación en Didáctica de la Matemática, Vol. 1(3), 99-111.
Recuperado de http://revistaseug.ugr.es/index. php/pna /article/view/6212

Niss, M. (2002). Mathematical competencies and the learning of mathematics: The danish KOM proyect. Roskilde: Roskilde University. Recuperado de

http://www.math.chalmers.se/Math/Grundutb/ $\mathrm{CTH} / \mathrm{mve} 375 / 1112 /$ docs/KOMkompetenser. pdf

Paredes, J.D. y Sanabria, W.M. (2015). Ambientes de aprendizaje o ambientes educativos. Una reflexión ineludible. Revista de Investigaciones UCM, 15(25): 144-158

Parra, O., y Díaz, V. (2014). Didáctica de las matemáticas y tecnologías de la información y la comunicación. Revista Educación y Desarrollo Social, Vol. 8(2), 60-81. Recuperado de https:// revistas.unimilitar.edu.co/index.php/reds/ article/view/295/101

Perrenoud, P. (2000). Construire des compétences dans l'école. París: ESF. Recuperado de https:// www.unige.ch/fapse/SSE/teachers/perrenoud/ php_main/php_livres/php_co nstruire.html

Rico, L., y Lupiañez, J. (2009). Competencias matemáticas desde una perspectiva curricular. Revista de Currículum y Formación de Profesorado, 12(3), 28-31. Recuperado de https://recyt.fecyt.es/index. php/profesorado/ article/download/43579/25488

Tobón, S. (2013). Formación integralycompetencias. Pensamiento complejo, currículo, didáctica y evaluación. Colombia: ECOE Ediciones 